\title{
Antibiotic prophylaxis in otolaryngologic surgery
}

\section{Ana Carolina Xavier Ottoline', Shiro Tomita², Marise da Penha Costa Marques 3 , Felippe Felix 3 , Priscila Novaes Ferraiolo Roberta Silveira Santos Laurindo4.}

\author{
1) Doctor (Resident) \\ 2) Professor (Staff). \\ 3) Master Degree (Physician Assistant) \\ 4) Doctor. \\ Institution: Hospital Universitário Clementino Fraga Filho, Universidade Federal do Rio de Janeiro \\ Rio de Janeiro / RJ - Brazil. \\ Mailing address: Ana Carolina X. Ottoline - Rua Professor Rodolpho Paulo Rocco, 255, 11ªndar - Ilha do Fundão - Rio de Janeiro / RJ - Brazil - Zip Code: 21941 - 913 \\ Telephone: (+55 21) 2562-2842 - E-mail: carol_ottoline@yahoo.com.br \\ Article received on May 24, 2012. Article accepted on September 24, 2012.
}

\section{SUMMARY}

Aim: Antibiotic prophylaxis aims to prevent infection of surgical sites before contamination or infection occurs. Prolonged antibiotic prophylaxis does not enhance the prevention of surgical infection and is associated with higher rates of antibioticresistant microorganisms. This review of the literature concerning antibiotic prophylaxis, with an emphasis on otolaryngologic surgery, aims to develop a guide for the use of antibiotic prophylaxis in otolaryngologic surgery in order to reduce the numbers of complications stemming from the indiscriminate use of antibiotics.

Keywords: Antibiotic Prophylaxis; Otorhinolaryngologic Surgical Procedures; Infection.

\section{INTRODUCTION}

Surgical antibiotic prophylaxis is defined as the administration of an antimicrobial agent prior to contamination of previously sterile spaces and fluids (1). The purpose of this procedure is to eradicate transient organisms with the potential to colonize and slow the growth of resident microbes, thus preventing surgical infection (1). Antibiotic prophylaxis should be performed in conjunction with any surgery associated with a high risk for infection or when the consequences of infection would be disastrous (even if the risk for infection is low) (2).

According to the classification scheme of the American National Academy of Science and the National Research, surgical wounds are classified as clean injuries (no violation of septic technique, no manipulation of the gastrointestinal tract or genitourinary, or respiratory system, and no sign of inflammation), clean-contaminated injuries (manipulation of the digestive or respiratory tract and/or minimal violation of sterile technique), contaminated injuries (active inflammation and/or violation of sterile technique), or dirty injuries (purulent inflammation) (3).

The choice of antibiotic is influenced by the need for the drug to be administered intravenously to be effective against agents that might colonize the surgical site and to be bactericidal, achieve adequate tissue levels, cause minimal side effects, be relatively cost-effective (half-life), and have a minimal impact on the local colonizers of the patient and the hospital (4). Cefazolin meets all of these requirements and is thus the first choice for surgical prophylaxis for clean-contaminated head and neck surgeries according to the recommendations of the Antimicrobial Agents Committee of the Surgical Infections Society and American Society of Health-System Pharmacists of 1999. Clindamycin should be used in patients allergic to beta-lactams. Amoxicillin-clavulanate is the first choice for surgeries with risk for contamination by anaerobes (4).

Antibiotic prophylaxis should be initiated during anesthesia. The dose should be repeated only if the surgical time exceeds the half-life of the antibiotic or if major bleeding (10-20\% of the blood volume) occurs. Prolonged antibiotic prophylaxis does not enhance the prevention of surgical infection and is associated with higher levels of bacterial resistance (5). Furthermore, the indiscriminate use of antibiotics can lead to serious complications such as toxic reactions, reduces the stimulus to antibody formation, and is financially burdensome (6).

This article will discuss the main aspects of antibiotic prophylaxis, with an emphasis on otolaryngologic surgeries, and its main objective is to develop a guide for the use of antibiotic prophylaxis in otolaryngologic surgeries in order to reduce the complications of the indiscriminate use of antibiotics. 


\section{ReVIeW}

\section{Pharyngeal surgeries}

The most common pharyngeal colonizers are Grampositive cocci, mainly Peptostreptococcusand Peptococcus spp., and anaerobic bacteria $(4,7)$. In the oropharynx, anaerobes are 10 times more numerous than aerobes (7). Gram-negative bacteria are rare in the secretions of healthy individuals; nevertheless, organisms such as Klebsiella, Pseudomonas, Proteus, and some Bacteroides species (other than B. fragilis) are common colonizers of the aerodigestive tracts of oncology patients (8).

\section{Adenotonsillectomy}

Adenotonsillectomy has high immediate postoperative morbidity, including sore throat, dysphagia, fever, halitosis, weight loss, and reluctance to take food orally (9.10).

Colonization of the open tonsillar fossa by the oropharyngeal flora has been suggested to produce a local inflammatory response that exacerbates postoperative pain (9). This idea inspired several studies of the relationship between the perioperative use of antibiotics and postoperative morbidity (11).

According to OBESO S et al. in 2010 (12), the administration of antibiotics in pediatric patients significantly reduced the early return to normal diet (level of evidence A), did not reduce pain or the risk for bleeding (level A) and did not provide major benefits over the postoperative application of topical antibiotics (level A), while topical antibiotics were more effective than systemic antibiotics at reducing pain (level B).

Although the findings of individual studies vary, there is no evidence to support a consistent, clinically important, beneficial effect of antibiotics on the major morbid outcomes of tonsillectomy. The limited benefit of antibiotics observed may have resulted from a positive bias introduced by several important methodological shortcomings in the included trials. Therefore, based on existing evidence, we would advocate against the routine prescription of antibiotics to patients undergoing tonsillectomy (13).

The only established reason for antibiotic prophylaxis in tonsillectomy is to prevent endocarditis and sepsis in patients with orthopedic implants or prosthetic valves or a history of previous endocarditis, congenital heart disease, or transplant with valvulopathy (11).
Uvulopalatopharyngoplasty

There are no controlled studies showing a benefit or necessity of perioperative antibiotic administration. As the procedure involves an incision in the pharyngeal mucosa, with the consequent risk for infection by anaerobes, we recommend the use of prophylactic antibiotics in this surgery (14).

\section{Rhinologic surgeries}

Even healthy individuals have potentially pathogenic species such as S. aureus, Klebsiella spp., and Escherichia coli in their nostrils in 77\% of cases. Between 18\% and 50\% of patients are colonized by $S$. aureus (15).

Septoplasty

There are few studies demonstrating the necessity or effectiveness of antibiotic prophylaxis in nasal surgery. Many authors therefore consider it unnecessary (6).

Surgical procedures on the aerodigestive tract are considered potentially contaminated and may be associated with postoperative infectious complications such as toxic shock syndrome, osteomyelitis, meningitis, and cavernous sinus thrombosis (6). Bacteremia can lead to endocarditis in at-risk patients, such as patients with valvular prostheses, cardiac transplants, or histories of endocarditis. An antibiotic is recommended in such cases $(16,17)$.

Staphylococcal toxic shock syndrome is extremely rare, with an estimated incidence of $0.0002 \%$, but there is evidence that it can be prevented by the prophylactic use of antibiotics. There are no studies showing the incidence of bacteremia when nasal packing antibiotics are used (12).

Scientific evidence shows that systemic administration of antibiotics does not reduce morbidity or infection after septoplasty (level A), antibiotics do not protect against $S$. aureus colonization and do contribute to decreased levels of normal flora (18), antibiotics do not seem to confer beneficial flora changes (18), and topical antibiotics decrease colonization of nasal septoplasty sites (level A) (12).

No studies showed a benefit of the perioperative use of systemic antibiotics in septoplasty (6). Therefore, septal surgery does not require routine antibiotic prophylaxis because of the low risk for infection (19).

\section{Rhinoplasty and other nasal surgeries}

Antibiotic prophylaxis is not necessary in primary rhinoplasty without grafting or septoplasty. 
In complex nasal surgeries, such as secondary septorhinoplasty, nasal graft surgery, or the repair of septal defects, the infection rate reaches $27 \%$ (20). These patients might benefit from the use of antibiotics, but there are no studies comparing the perioperative use of antibiotics versus placebo (20). Although the benefits of prophylactic antibiotics for this type of indication remain uncertain, most authors recommend their use in such cases (20).

\section{Laryngeal surgeries}

Isolates of bacteria from infected clean-contaminated head and neck surgery wounds commonly have polymicrobial characteristics. Gram-positive bacteria are most frequently isolated, followed by Gram-negative species and anaerobes (21).

\section{Laryngeal microsurgery}

The infection rate is low, and there are no clinical trials showing a benefit of antibiotic use in these surgeries.

\section{Other laryngeal surgeries}

Antibiotic therapy is necessary in complex laryngeal surgeries such as total laryngectomy. The presence of potentially pathogenic bacteria, the duration of the procedure, and the use of flaps lead to a high rate of infection in the absence of antibiotic (22).

\section{Endoscopic sinus surgery}

There are potentially pathogenic species such as $S$. aureus, Klebsiella spp., and/or Escherichia coli in the nostrils in $77 \%$ of cases (23). In patients diagnosed with chronic rhinosinusitis, repeated antibiotic therapy has selected for resistant pathogenic species. Up to $90 \%$ of patients undergoing endoscopic sinonasal surgery produce positive cultures; most of the species isolated are resistant to penicillin, and 65\% are resistant to cephalosporins (24).

\section{Endoscopic sinus surgery for chronic rhinosinusitis}

We suggest amoxicillin-clavulanate and prednisolone for 14 days, beginning 7 days before surgery, for the treatment of chronic infectious processes and reduction of intraoperative complications (25) and to improve the postoperative results. Antibiotic prophylaxis is recommended during anesthesia.

Endoscopic surgery for closure of cerebrospinal fluid fistulae

Perioperative systemic administration of antibiotics reduces the incidence of postoperative infection (12).

\section{Endoscopic sphenopalatine artery ligation}

Antibiotics are not recommended for this procedure because it is considered a clean surgery (3).

\section{Dacryocystorhinostomy}

Infection occurs after $8 \%$ of surgeries that open the lacrimal sac. Antibiotic administration can reduce this rate by up to 5 -fold (26), justifying the use of antibiotic prophylaxis.

Resection of nasal tumors by an external approach

Antibiotics are recommended because of the high rate of infectious complications (27).

\section{Otologic surgeries}

Postoperative infection in otology manifests as loss of the neotympanic graft, labyrinthitis, surgical wound infection, or the occurrence of otitis media or external otitis.

VERSCHUUR et al. (28) have proposed the following classification scheme: clean surgery: myringoplasty, stapedectomy, ossicular reconstruction, and dry ears; cleancontaminated or dirty surgery: ears with preoperative suppuration (chronic otitis media with or without cholesteatoma).

The Pseudomonasspecies isolated from the ear are highly sensitive to polymyxin $\mathrm{B}$, ciprofloxacin, and gentamicin, while the Staphylococcusspecies are sensitive to cloxacillin, gentamicin, and ciprofloxacin. Most isolates obtained during episodes of otorrhea are polymicrobial. The microbiological profile of otitis media with cholesteatoma is similar to that of simple chronic otitis media except that anaerobes, mainly Bacteroides and Peptococcus, are more frequent; anaerobes have been isolated in up to two-thirds of cases of otorrhea with cholesteatoma. Although otitis media with effusion is assumed not to present with active infection, microorganisms, most frequently Haemophilus influenza, Moraxella catarrbalis, and Streptococcus pneumoniae, are isolated in up to $50 \%$ of cases.

The incidence of postoperative infection has been estimated at less than $5 \%$ after clean surgery but between 7 and 14\% after clean-contaminated surgery (29). The most common infectious agents encountered after clean otologic surgery are species of $S$. aureus and other Grampositive bacteria (30). The bacteria most frequently isolated from cases of chronic otitis media without cholesteatoma are Pseudomonas aeruginosa and Staphylococcusspecies, 
mainly $S$. aureus. The other Gram-negative organisms encountered are, in order of frequency, Klebsiella, Proteus, and Haemophilus; the remaining Gram-positive bacteria are predominantly Streptococci, and the isolation of anaerobes is uncommon (31).

Chronic otitis media with cholesteatoma differs from that without in that anaerobes, especially Bacteroides and Peptococcus, are the most common isolates; otherwise, the bacteriological profile is similar (28).

Although serous otitis media involves no active infection, microorganisms, most frequently Haemophilus influenzae, M. catarrbalis, and Streptococcuspneumoniae, are isolated in 50\% of cases (31).

According to OBEso et al. in 2010 (12), systemic administration of antibiotics does not reduce the incidence of infection after clean (level A) or cleancontaminated (level B) surgery and provides no benefit over topical antibiotic treatment in clean-contaminated surgery (level B); perioperative systemic administration of antibiotics did reduce infection in cases of cleancontaminated surgery with positive preoperative cultures (level B).

Tympanoplasty

There is no evidence that perioperative systemic administration of antibiotics decreases the incidence of infection (12).

\section{Mastoidectomy}

Antibiotic use is always recommended. In cases with perioperative otorrhea, antibiotics should be administered post-operatively (12).

\section{Stapedotomy}

There is no evidence to recommend the perioperative use of systemic antibiotics in patients undergoing clean otologic surgery (28). However, antibiotic prophylaxis is recommended in cases in which a surgical infection could evolve with serious consequences, such as deafness or labyrinthitis.

\section{Cochlear Implants}

Cochlear implant surgery is considered a clean surgery, and there are no randomized studies comparing the incidence of local infection between patients with and without antibiotic treatment (11). Perioperative systemic administration of antibiotics has been suggested to reduce the incidence of meningitis (12).

\section{Ossicular Reconstruction}

Ossicular reconstruction is considered a clean surgery (28). Perioperative systemic administration of antibiotics is not recommended because the incidence of infection is low (12).

Otoplasty

Otoplasty is considered a clean surgery. Careful asepsis and antisepsis are essential for preventing infection. Infection of either the skin or subcutaneous tissue or perichondritis is unusual after otoplasty (26).

Insertion of transtympanic drainage tubes

According to Verschuur et al., ears without effusion are considered clean, those with seromucous effusion clean-contaminated, and those with purulent effusion dirty (28). Several well-designed studies have shown no significant difference in the incidence of otorrhea between patients treated postoperatively with oral or topical antibiotics. As the efficacy of intraoperative irrigation with saline solution was confirmed in another randomized clinical trial, there is a level of evidence of " $\mathrm{A}$ " to recommend washing the ear with this solution (12).

\section{Surgery on the salivary glands}

Some surgeries, such as parotidectomy and resection of the submandibular glands, are considered clean (3). Antibiotic prophylaxis is not routinely recommended in such cases (32).

\section{DISCUSSION}

Most otolaryngologic surgeries are considered potentially contaminated, and prophylaxis is recommended in some cases. In consideration of the principles of antibiotic prophylaxis and the particularities of each surgical procedure, we make the following recommendations.

\section{General Recommendations}

1. Indications: Antibiotic prophylaxis is indicated in surgeries in which complications are frequent or severe and studies show the effectiveness of this use and also in procedures considered high risk but for which there are insufficient data to prove the effectiveness of prophylaxis.

2. Best time for antibiotic prophylaxis: About 30 minutes before the incision, usually coinciding with the induction of anesthesia in order to ensure adequate 
Table I. Suggestions for antibiotic prophylaxis in otolaryngologic surgery.

\begin{tabular}{|c|c|c|c|c|}
\hline Antibiotic & $\begin{array}{l}\text { Initial dose } \\
\text { (induction) }\end{array}$ & $\begin{array}{l}\text { Additional doses } \\
\text { duringsurgery }\end{array}$ & Dose inchildren & $\begin{array}{l}\text { Dose in patients } \\
\text { over } 90 \mathrm{~kg}\end{array}$ \\
\hline Cefazolin & $2 g$ & I gevery $3 \mathrm{~h}$ & $\begin{array}{l}50 \mathrm{mg} / \mathrm{kg} \text { for induction } \\
\text { Half of the initial dose every } 3 \mathrm{~h}\end{array}$ & $\begin{array}{l}3 \text { gforinduction } \\
1.5 \text { gevery } 3 \mathrm{~h}\end{array}$ \\
\hline $\begin{array}{l}\text { Amoxicillin + sulbactam } \\
\text { orAmoxicillin-clavulanate }\end{array}$ & $1.5 \mathrm{~g}$ & I gevery $3 \mathrm{~h}$ & $\begin{array}{l}40 \mathrm{mg} / \mathrm{kg} \text { for induction } \\
\text { Half of the initial dose every } 3 \mathrm{~h}\end{array}$ & $\begin{array}{l}2 \text { gforinduction } \\
1.5 \text { gevery } 3 \mathrm{~h}\end{array}$ \\
\hline Clindamycin & $600 \mathrm{mg}$ & None necessary & $\begin{array}{l}30 \mathrm{mg} / \mathrm{kg} \text { up to } 600 \mathrm{mg} \text { for induction } \\
\text { The minimum dose must be } 300 \mathrm{mg} \\
\text { regardless ofbody weight }\end{array}$ & 900 mg for induction \\
\hline
\end{tabular}

Note: 1 - Clindamycin should be reserved for patients allergic to beta-lactams.

2 - Amoxicillin-clavulanate is recommended for chronic otitis media.

Table 2. Antibiotic prophylaxis in otolaryngologic surgery

When should it be used?
Endoscopic sinus surgery for chronic rhinosinusitis
Nasalgraftsurgery
Dacryocystorhinostomy
Stapedotomy
Endoscopic surgery for closure of cerebrospinal fluid fistulae
Cochlear implants
Totallaryngectomy
Mastoidectomy
Repair of septal defects
Resection of nasaltumors by an external approach
Reviewseptorhinoplasty
Uvulopalatopharyngoplasty

serum and tissue drug concentrations at the time of tissue damage.

3. Recommended doses: The recommended initial dose is the maximum indicated for each drug. Protective plasma levels must be maintained throughout the surgery. The dose should be repeated during surgery according to the half-life of the chosen antibiotic.

4. Duration of prophylaxis: There is no advantage to prolonging prophylaxis beyond the surgical time in the majority of patients.

5. Patients already receiving antibiotics: When the patient has an infection that requires antibiotic treatment and also requires surgical intervention, we recommend a dose adjustment to ensure that the next administration time is during the surgery and that the tissue drug concentration is maintained throughout the procedure. The antibiotic can be continued postoperatively for a variable period according to the details of the individual case.

6. Prolonged antibiotic prophylaxis is not indicated for accidental contamination of the surgical site.

7. With the exception of patients with an infection (i.e., those for whom the prescription of antibiotics has a

When should it not be used?
Adenotonsillectomy
Insertion oftranstympanic drainage tubes
Endoscopic sphenopalatine artery ligation
Lymphadenectomy
Laryngeal microsurgery
Otoplasty
Parotidectomy
Ossicular reconstruction
Primaryseptorhinoplasty
Septoplasty
Resection ofthe submandibularglands
Tympanoplasty

therapeutic purpose), oral antibiotics should not be prescribed after discharge. (Table 1 and 2)

\section{FINAL COMMENTS}

Antibiotic prophylaxis in otolaryngologic surgery remains a controversial topic that requires further study. Based on this discussion, we propose a guide for uniform procedures in order to reduce the damage caused by the misuse of antibiotics.

\section{REFERENCES}

1. Fennessy BG, Harney M, O'Sullivan MJ, Timon G. Antimicrobial prophylaxis in otorhinolaryngology/head and neck surgery. Clin Otolaryngol. 2007;32:204-7.

2. Fairbanks DN. In: Bayley BJ, Calhoun KH et al. Head and Neck Otolaryngology. Third edition. Philadelphia, Lippincott: Williams\& Wilkins; 2001. p.47-54.

3. Tandon JT \& Jonson JT. Wound infection in head and 
neck surgery: prophylaxis, etiology and management. J Otolaryngol. 1990;19:197-200.

4. American Society of Health-System Pharmacists. ASHP therapeutic guidelines on antimicrobial prophylaxis in surgery. Am J Health Syst Pharm. 1999;56:1839-88.

5. Strong MT. Wound infection in otolaryngologic surgery and the inexpediency of antibiotic prophylaxis. Laryngoscope. 1963;73(2):165-84.

6. Caniello M, Passerotti GH, Goto EY, Voegels RL, Butugan O.Antibiotics in septoplasty: is it necessary? Rev Bras Otorrinolaringol. 2005;71(6):734-8.

7. Weber RS \& Callender DL. Antibiotic prophylaxis in cleancontaminated head and neck oncologic surgery. Ann Otol Rhinol Laryngol Suppl. 1992;155:16-20.

8. Bartlett G \& Gorbach SL. Anaerobic infections of the head and neck. Otolaryngol Clin North Am. 1976;9:65578.

9. Mann ER, Blair EA, Levy AJ, Chang A. Effect of topical antibiotic therapy on recovery after tonsillectomy in adults. Otolaryngology-Head and Neck Surgery. 1999;121(3):27782.

10. Grandis J et al. The efficacy of perioperative antibiotic therapy on recovery following tonsillectomy in adults: Randomized doubleblind placebo controlled trial. Otolaryngology - Head and Neck Surgery. 1992;106(2):13742.

11. Piltcher OB, Scarton FB.Antibiotics in tonsillectomies: therapeutic or prophylactic? Necessary or outrageous? Rev Bras Otorrinolaringol. 2005;71(5):686-90.

12. Obeso Set al. Antibiotic prophylaxis in otolaryngologic surgery. Acta Otorrinolaringol Esp. 2010;61(1):54-68.

13. Dhiwakar M, Clement WA, Supriya, McKerrow W. Antibiotics to reduce post-tonsillectomy morbity. Cochrane Database Syst Rev. 2010 Jul7;(7):CD005607.

14. Antimicrobial prophylaxis for surgery. Treat Guidel Med Lett 2009;7:47.

15. Gluck U \& Gebbers JO. The nose as bacterial reservoir: important differences between the vestibule and cavity. Laryngoscope. 2000;110:426-8.

16. Kaygusuz I, Kizirgil A, KarlidagT, Yalcin S, Keles E, Yakupogullri Y, et al. Bacteriemia in septoplasty and septorhinoplasty surgery. Rhinology. 2003;41:76-9.
17. JayawardenaS, EisdorferJ, IndulkarS, Zarkaria M. Infective endocarditis of native valve after anterior nasal packing. Am J Ther. 2006;13:460-2.

18. Karaman E, Alimoglu Y, Aygun G, Kilic E, Yagiz C. Effect of septoplasty and per-operative antibiotic prophylaxis on nasal flora. B-ENT. 2012;8(1):13-9.

19. Ricci G, D'Ascanio L. Antibiotics in septoplasty: evidence or habit?. Am J Rhinol Allergy. 2012 MayJun;26(3):194-6.

20. Andrews PJ, East CA, Jayaraj SM, Badia L, Panagamuwa C, Harding L. Prophylactic vs postoperative antibiotic use in complex septorhinoplasty surgery. Arch Facial Plast Surg. 2006;8:84-7.

21. Rodrigo JP, AlvárezJC, Gómez JR, Suárez C, Fernández JA, MartinezJA. Comparison of three prophylactic antibiotic regimens in clean-contaminated head and neck surgery. Head Neck. 1997;19:188-93.

22. Dedivitis RA, Guimarães AV: Antibiotic prophylaxis in head and neck cancer surgery. Acta Medica Misericordiæ. 2000;3(1):28-31

23. Gluck U, Gebbers JO. The nose as bacterial reservoir: important differences between the vestibule and cavity. Laryngoscope. 2000;110:426-8.

24. Shikani AH. Use of antibiotics for expansion of the Merocel parking following endoscopic sinus surgery. Ear Nose Throat J. 1996;75:524-6.

25. Hassan H, Ramadan, MD. Corticosteroid therapy during endoscopic sinus surgery in children. Arch Otolaryngol Head Neck Surg. 2001;127(2):188-192.

26. Neto SC, Mello JFJ, Martins RHG, Costa SS. Tratado de Otorrinolaringologia e Cirurgia Cérvico Facial, 2ed. SãoPaulo. Roca, 2011.

27. Santos LRM. Complications in surgery of the nasal cavity and paranasal sinuses. Rev Bras Cir Cab Pesc. 2003;31(2):2325.

28. Verschuur HP, De Wever WWH, Van Benthem PPG. Antibiotic prophylaxis in clean and clean-contaminated ear surgery. Cochrane Database Syst Rev. 2004 CD003996.

29. Palva T, Palva A, Salmivalli A. Radical mastoidectomy with cavity obliteration. Arch Otolaryngol. 1968;88:119-23.

30. Jackson CG. Antimicrobial prophylaxis in ear surgery. Laryngoscope. 1988; 98:1116-23. 
31. Fairbanks DNF. Antimicrobial therapy in otolaryngologyhead and neck surgery. 8th ed. Alexandria: American Academy of Otolaryngology-Head and Neck Surgery Foundation; 1996. p.47-50.
32. ManLX, Beswick DM, JohnsonJT. Antibiotic prophylaxis in uncontaminated neck dissection. Laryngoscope. 2011 Jul;121(7):1473-7. 